\title{
On Relay Selection for Decode-and-Forward Relaying
}

\author{
Muhammad Mehboob Fareed, Student Member, IEEE, and Murat Uysal, Senior Member, IEEE
}

\begin{abstract}
In this letter, we consider a multi-relay network operating in decode-and-forward mode. We propose a novel relay selection method with a low implementation complexity. Unlike the competing schemes, it requires neither error detection methods at relay nodes nor feedback information at the source. We derive a closed-form symbol error rate (SER) expression for multi-relay network under consideration and demonstrate that the proposed selection method is able to extract the full diversity. Extensive Monte Carlo simulations are also presented to confirm the derived SER expressions and to compare the performance of the proposed scheme with its competitors.
\end{abstract}

Index Terms-Distributed space-time codes, pairwise error probability, power allocation, relay channels.

\section{INTRODUCTION}

$\mathbf{E}$ XPLOITING broadcasting nature in wireless transmission, cooperative diversity [1-3] creates a virtual antenna array among cooperating nodes and extracts spatial diversity advantages in a distributed manner. The cooperation can take place either with regenerative (decode-and-forward) or non-regenerative (amplify-and-forward) relaying techniques. Digital processing nature of decode-and-forward $(\mathrm{DaF})$ relaying makes it more practical than amplify-and-forward (AaF) relaying which requires expensive RF transceivers.

For $\mathrm{DaF}$ relaying in a single-relay scenario, Sendonaris et al. [2] have presented a maximum likelihood (ML) decoder and demonstrated that it is able to provide full diversity. Since the complexity of this detector becomes unmanageable for higher order modulations, so-called $\lambda$-MRC decoder has been proposed in [3]. $\lambda$-MRC decoder is a variant of maximum ratio combining (MRC) and relies on source-to-relay channel state information (CSI) to construct a weighted MRC metric. In [4], Laneman et al. have shown that full diversity in DaF relaying can be achieved with conventional MRC if relay node(s) only forward the correctly decoded information. The practical implementation of such an approach requires the use of error detection methods such as cyclic redundancy check (CRC) at the relay terminal. In [5], Wang et al. have presented a demodulation scheme called cooperative MRC (CMRC) which achieves full diversity without the use of CRC. However, their proposed method needs CSI of all underlying links at destination node to construct MRC weights and suffers from low throughput.

Manuscript received November 20, 2007; revised June 21, 2008 and December 6, 2008; accepted February 19, 2009. The associate editor coordinating the review of this letter and approving it for publication was Y. J. (A.) Zhang.

The authors are with the Department of Electrical and Computer Engineering, University of Waterloo, Waterloo, ON, N2L3G1, Canada (e-mail: $\{$ mmfareed, muysal $@ @$ ece.uwaterloo.ca).

This paper was presented in part at Canadian Conference on Electrical and Computer Engineering , May 2008 [1]. The work of M. Uysal is supported in part by a Natural Sciences and Engineering Research Council of Canada Special Opportunity Grant (SROPJ305821-05).

Digital Object Identifier 10.1109/TWC.2009.071306
In contrast to earlier works which assume the participation of all relays, relay selection has emerged as a powerful technique with a higher throughput, because fewer time slots are required to complete transmission of one block. In [6], Bletsas et al. have proposed a simple relay selection criterion for a multi-relay network. Their method first searches the set of relays which are able to decode successfully (i.e., practical implementation requires error detection such as CRC) and then chooses the "best" relay for transmission in relaying phase. Determination of the best relay depends either on the minimum or harmonic mean of source-to-relay and relayto-destination channel signal-to-noise ratios (SNRs). In [7], Beres and Adve have proposed another selection criterion in which relay-to-destination link with the maximum SNR is chosen. In [8], Ibrahim et al. have introduced a relay selection method based on the scaled harmonic mean of instantaneous source-to-relay and relay-to-destination channel SNRs. This close-loop scheme requires feedback of sourceto-relay and relay-to-destination CSIs to the source node so that power can be adjusted before transmission. An error rate performance analysis is further presented in [8] which is mainly restricted for a symmetrical case where relay nodes are located equidistant from the source. A recently proposed scheme by Yi and Kim [9] combines relay selection with CMRC. They also consider link adaptive regeneration (LAR) [10] where decoded symbols at relay nodes are scaled in power before being forwarded to the destination. LAR method results in reduced signaling overhead as compared to C-MRC.

In this paper, we investigate the performance of a multirelay network with relay selection avoiding some restrictions and assumptions imposed in previous works. We propose a relay selection criterion based on an open-loop architecture. It does not require any feedback CSI unlike [8] which relies on power allocation by the source node through feedback information. It further does not require any error detection mechanism (e.g., CRC) at relay nodes in contrast to [6], [7]. In our scheme, the destination node chooses the best relay based on the minimum of source-to-relay and relay-to-destination SNRs at the end of broadcasting phase and allows the selected relay to participate only if the minimum of its source-to-relay and relay-to-destination link SNRs is greater than SNR of the direct link. We derive closed-form symbol error rate (SER) performance expressions for the multi-relay network scenario with the proposed relay selection algorithm. In our analysis, we assume arbitrary relay locations, thereby avoiding the symmetrical scenario of [8] which is a simplifying assumption, yet somewhat impractical in real-life situations. Extensive Monte-Carlo simulations are also presented to collaborate on the analytical results.

The rest of the paper is organized as follows: In Section II, we describe the multi-relay cooperative network under 
consideration with $\mathrm{DaF}$ relaying and relay selection. In Section III, we derive SER for multi-relays with arbitrary locations. In Section IV, we present simulation results and finally, Section $\mathrm{V}$ concludes the paper.

Notation: $E_{y}($.$) denotes expectation with respect to random$ variable $y$.

\section{TRANSMISSION MODEL}

We consider a multi-relay scenario with $N$ relay nodes. Source, relay, and destination nodes operate in half-duplex mode and are equipped with single transmit and receive antennas. All the nodes are assumed to be located in a twodimensional plane where $d_{S D}, d_{S R_{i}}$, and $d_{R_{i} D}, i=1,2, \ldots, N$ denote the distances of source-to-destination $(\mathrm{S} \rightarrow \mathrm{D})$, sourceto-relay $\left(\mathrm{S} \rightarrow \mathrm{R}_{i}\right)$, and relay-to-destination $\left(\mathrm{R}_{i} \rightarrow \mathrm{D}\right)$ links respectively. We consider an aggregate channel model which takes into account both long-term path loss and short-term Rayleigh fading. The path loss is proportional to $d^{-\alpha}$ where $d$ is the propagation distance and $\alpha$ is path loss coefficient. Normalizing the path loss in $\mathrm{S} \rightarrow \mathrm{D}$ to be unity, the relative geometrical gains of $\mathrm{S} \rightarrow \mathrm{R}_{i}$ and $\mathrm{R}_{i} \rightarrow \mathrm{D}$ links are defined as $G_{S R_{i}}=\left(d_{S D} / d_{S R_{i}}\right)^{\alpha}$ and $G_{R_{i} D}=\left(d_{S D} / d_{R_{i} D}\right)^{\alpha}$. They can be further related to each other by law of cosines, i.e., $G_{S R_{i}}^{-2 / \alpha}+G_{R_{i} D}^{-2 / \alpha}-2 G_{S R_{i}}^{-1 / \alpha} G_{R_{i} D}^{-1 / \alpha} \cos \theta_{i}=1$, where $\theta_{i}$ is the angle between lines $\mathrm{S} \rightarrow \mathrm{R}_{i}$ and $\mathrm{R}_{i} \rightarrow \mathrm{D}$ [11]. The fading coefficients for $\mathrm{S} \rightarrow \mathrm{D}, \mathrm{S} \rightarrow \mathrm{R}_{i}$, and $\mathrm{R}_{i} \rightarrow \mathrm{D}$ links are denoted by $h_{S D}, h_{S R_{i}}$, and $h_{R_{i} D}$, respectively and are modeled as zeromean complex Gaussian random variables with unit variance leading to a Rayleigh fading channel model.

Let $x$ be a modulation symbol taken from an M-PSK (Phase Shift Keying) constellation. Considering path-loss effects, the received signals in the first time slot at destination and at $i$ th relay nodes are given by

$$
\begin{gathered}
r_{D 1}=\sqrt{K_{S} P} h_{S D} x+n_{D 1}, \\
r_{R_{i}}=\sqrt{G_{S R_{i}} K_{S} P} h_{S R_{i}} x+n_{R_{i}},
\end{gathered}
$$

where $P$ is the total transmit power shared by the source and relay nodes. $K_{S}$ is an optimization parameter for power allocation and denotes the fraction of power used by the source node in the broadcasting phase. For equal power allocation, $K_{S}=0.5$. The remaining power is reserved for relay transmission and power of the selected relay is controlled by another optimization parameter, $K_{i}, i=1,2, \ldots N$. Optimized values of $K_{S}$ and $K_{i}, i=1,2, \ldots, N$ can be found in [1]. In (1)-(2), $n_{R_{i}}$ and $n_{D 1}$ model the additive noise terms and are assumed to be complex Gaussian with zero mean and variance of $N_{0}$.

Let $\lambda_{S D}, \lambda_{S R_{i}}$, and $\lambda_{R_{i} D}$ denote the instantaneous SNRs in $\mathrm{S} \rightarrow \mathrm{D}, \mathrm{S} \rightarrow \mathrm{R}_{i}$, and $\mathrm{R}_{i} \rightarrow \mathrm{D}$ links respectively. In our scheme, the destination first chooses the best relay based on the following criterion

$$
R_{\text {sel }}=\arg \max _{R_{i}}\left\{\min \left(\lambda_{S R_{i}}, \lambda_{R_{i} D}\right)\right\},
$$

where "sel" denotes the index for the selected relay. Then, the destination node instructs the selected relay to participate in cooperation phase only if SNR in direct link is less than the minimum of the SNRs in the selected relaying path, i.e.,

$$
\lambda_{S D}<\lambda_{\max } \hat{=} \min \left(\lambda_{S R_{s e l}}, \lambda_{R_{s e l} D}\right) .
$$

Otherwise, the selected relay node will not participate in cooperation phase. If allowed to cooperate, the relay node performs demodulation ${ }^{1}$ and transmits re-encoded symbol $\hat{x}$ in the second time slot. The signal received at destination node is therefore given by

$$
r_{D 2}=\sqrt{G_{R_{s e l} D} K_{s e l} P} h_{R_{s e l} D} \hat{x}+n_{D 2},
$$

where $n_{D 2}$ models the additive Gaussian noise term and $K_{\text {sel }}=\left.K_{i}\right|_{i=s e l}$. The destination node then combines the received signals given by (1) and (5) using MRC and decodes the symbol transmitted by source.

\section{SER DERIVATION}

In this section, we derive a SER expression for the proposed scheme. Defining $\lambda=\left[\begin{array}{llll}\lambda_{S D} & \lambda_{\max } & \lambda_{S R_{s e l}} & \lambda_{R_{s e l} D}\end{array}\right]^{\mathrm{T}}$, a conditional SER expression can be given as

$$
P(e \mid \lambda)=P_{n-\text { coop }} P_{e \mid \text { direct }}+P_{\text {coop }} P_{e \mid c o o p},
$$

where $P_{n-\text { coop }}=P\left(\lambda_{S D}>\lambda_{\max }\right)$ is the probability that the selected relay is not qualified to participate in cooperation phase and $P_{\text {coop }}=1-P_{n-\text { coop }}$ is the probability of cooperation. $P_{\text {e|direct }}$ denotes the $\mathrm{SER}$ for direct $\mathrm{S} \rightarrow \mathrm{D}$ transmission and $P_{e \mid c o o p}$ denotes the SER when the cooperation takes place.

If cooperation does not take place, the overall SER is simply equal to the SER of direct link and is given by $P_{e \mid \text { direct }}=$ $\beta\left(\lambda_{S D}\right)$ where $\beta($.$) is given by [12]$

$$
\beta(x)=\frac{1}{\pi} \int_{0}^{(M-1) \pi / M} \exp \left(-\frac{g x}{\sin ^{2} \eta}\right) d \eta,
$$

with $g=\sin ^{2}(\pi / M)$. If cooperation takes place, we need to calculate $P_{\text {e|coop }}$ which is given by

$$
P_{e \mid c o o p}=P_{e_{-} s e l} P_{e \mid e_{-} s e l}+\left(1-P_{e_{-} s e l}\right) P_{e \mid c \_s e l},
$$

where $P_{e_{-} s e l}=\beta\left(\lambda_{S R_{s e l}}\right)$ denotes the probability of the selected relay to make a decoding error. If the selected relay makes an incorrect decision, the corresponding conditional SER is calculated as $P_{e \mid e_{-} s e l}=$ $\beta\left(\left|\lambda_{S D}+e \lambda_{R_{s e l} D}\right|^{2} /\left(\lambda_{S D}+\lambda_{R_{s e l} D}\right)\right)$. In the calculation of $P_{e \mid e_{-} s e l}$, we use $\hat{x}=e x$ to take into account for the error at the relay. We can actually approximate this probability by 1 , because, under the assumption that relay is qualified for cooperation (i.e., $\lambda_{\max }>\lambda_{S D}$ ), an incorrect decision at destination is much more likely than a correct one. On the other hand, if the selected relay has decoded correctly, the SER is given by $P_{e \mid e_{-} s e l}=\beta\left(\lambda_{S D}+\lambda_{R_{s e l} D}\right)$. Replacing all above related definitions in (6), we have

$$
\begin{aligned}
P(e \mid \lambda) & =\underbrace{P\left(\lambda_{S D}>\lambda_{\max }\right) \beta\left(\lambda_{S D}\right)}_{T_{0}} \\
& +\underbrace{P\left(\lambda_{S D}<\lambda_{\max }\right) \beta\left(\lambda_{S R_{s e l}}\right)}_{T_{1}} \\
& +\underbrace{P\left(\lambda_{S D}<\lambda_{\max }\right)\left[1-\beta\left(\lambda_{S R_{s e l}}\right)\right] \beta\left(\lambda_{R_{s e l} D}+\lambda_{S D}\right)}_{T_{2}} .
\end{aligned}
$$

\footnotetext{
${ }^{1}$ Although the relay basically performs demodulation, not decoding in our scheme, we still adopt the term decode-and-forward relaying following similar uses in the current literature, see e.g., [9].
} 
To find the unconditional SER, one needs to take an expectation of (9) with respect to $\lambda$. This is quite difficult and would probably not yield closed-form expressions. Therefore, we pursue an alternative approach here reformulating SER in terms of conditional probability density functions (pdfs) of $\lambda_{\max }, \lambda_{S R_{s e l}}$, and $\lambda_{R_{s e l} D}$ (conditioned on the event that $i$ th relay node is selected). Let $\xi_{i}, \xi_{i}^{C}, \mu_{i}$, and $\mu_{i}^{C}$ denote the events $\lambda_{\max }=\lambda_{S R_{i}}, \lambda_{\max }=\lambda_{R_{i} D}, \lambda_{S R_{i}}<\lambda_{R_{i} D}$, and $\lambda_{S R_{i}}>\lambda_{R_{i} D}$ respectively. Further, let $\Delta$ denote the set of permutations of $1,2, . ., N$ and $\operatorname{Pr}(\sigma)$ denote the probability of one particular permutation $\sigma \in \Delta . P_{e}$ can be then calculated as

$$
P_{e}=\sum_{i=1}^{N}\left[P\left(e \mid \xi_{i}\right) \operatorname{Pr}\left(\xi_{i}\right)+P\left(e \mid \xi_{i}^{C}\right) \operatorname{Pr}\left(\xi_{i}^{C}\right)\right],
$$

which can be further approximated as

$$
\begin{aligned}
P_{e} \cong \sum_{i=1}^{N}\left[\sum_{\sigma \in \Delta_{i}} \operatorname{Pr}(\sigma)\right] & {\left[\Phi_{0}+\sum_{k=1}^{2} \Phi_{k} \operatorname{Pr}\left(\mu_{i}\right)\right.} \\
& \left.+\sum_{k=1}^{2} \Phi_{k}^{C} \operatorname{Pr}\left(\mu_{i}^{C}\right)\right],
\end{aligned}
$$

where we define $\Phi_{k}=E_{\lambda}\left[T_{k}\right], k=0,1,2$ and ignore the correlation of $\mu_{i}$ and $\sigma$. Calculations of $\operatorname{Pr}(\sigma), \operatorname{Pr}\left(\mu_{i}\right)$, and $\operatorname{Pr}\left(\mu_{i}^{C}\right)$ are provided in Appendix A, while calculations of $\Phi_{0}, \Phi_{k}$, and $\Phi_{k}^{C}, k=1,2$ are given in Appendix B. Using these results from Appendixes, we obtain the final SER expression given on the top of next page, where $\Psi_{S R_{i}}($. and $\Psi_{R_{i} D}($.$) are the MGFs of \lambda_{S R_{i}}$ and $\lambda_{R_{i} D}$, respectively and $\alpha_{\eta_{k}}=-g / \sin ^{2} \eta_{k}, k=1,2$, . In the above, operators $F_{1}\{$.$\} and F_{2}\{$.$\} are defined by$

$$
\begin{gathered}
F_{1}\{f(\eta)\}=\frac{1}{\pi} \int_{0}^{\frac{(M-1) \pi}{M}} f(\eta) d \eta \\
F_{2}\left\{f\left(\eta_{1}, \eta_{2}\right)\right\}=\frac{1}{\pi^{2}} \int_{0}^{\frac{(M-1) \pi}{M}}\left[\int_{0}^{\frac{(M-1) \pi}{M}} f\left(\eta_{1}, \eta_{2}\right) d \eta_{1}\right] d \eta_{2} .
\end{gathered}
$$

We conclude this section by demonstrating the achievable diversity of our scheme. Assuming BPSK, inserting $\eta_{1}=\eta_{2}=$ $\pi / 2^{2}$, the resulting expression can be further approximated as

$$
\begin{aligned}
P_{e} \cong \frac{1}{2} \sum_{i=1}^{N}\left[\left\{(g \Lambda)^{-1}\left[\frac{\Lambda}{2} g\right]^{-N}\right\}\right. & \left\{1+\frac{N}{2}\left(1+(g \Lambda)^{-2}\right.\right. \\
& \left.\left.\left.-(g \Lambda)^{-2}(2)^{-N}\right)\right\}\right] .
\end{aligned}
$$

From (15), it can be readily confirmed that a diversity order of $N+1$ is achieved.

\section{NumericAl RESUlts AND Discussion}

In this section, we first verify the accuracy of derived SER expression through Monte-Carlo simulation. Then, we present performance comparisons between the proposed scheme and its competitors. We assume $\alpha=2, \theta_{i}=\pi$, and 4-PSK modulation in our simulation study.

\footnotetext{
${ }^{2}$ The value of $\eta_{1}=\pi / 2$ gives an upper bound for the integral in (13), but $\Phi_{0}, \Phi_{k}$, and $\Phi_{k}^{C}, k=1,2$ are the functions of sum and difference of integrals in the form of (13), (14) with different arguments. This makes the following result an approximation, not an upper or lower bound.
}

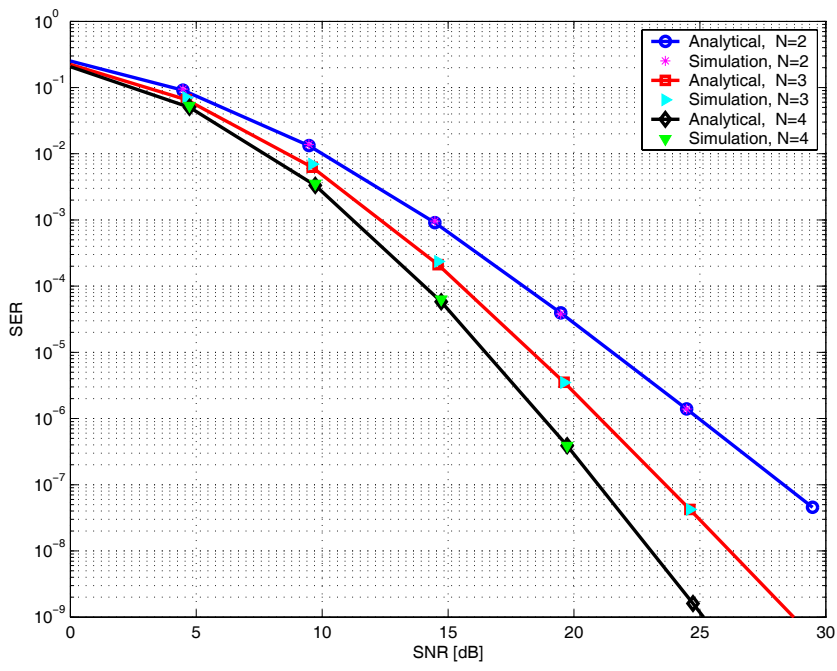

Fig. 1. Comparison of derived SER expression with simulation results.

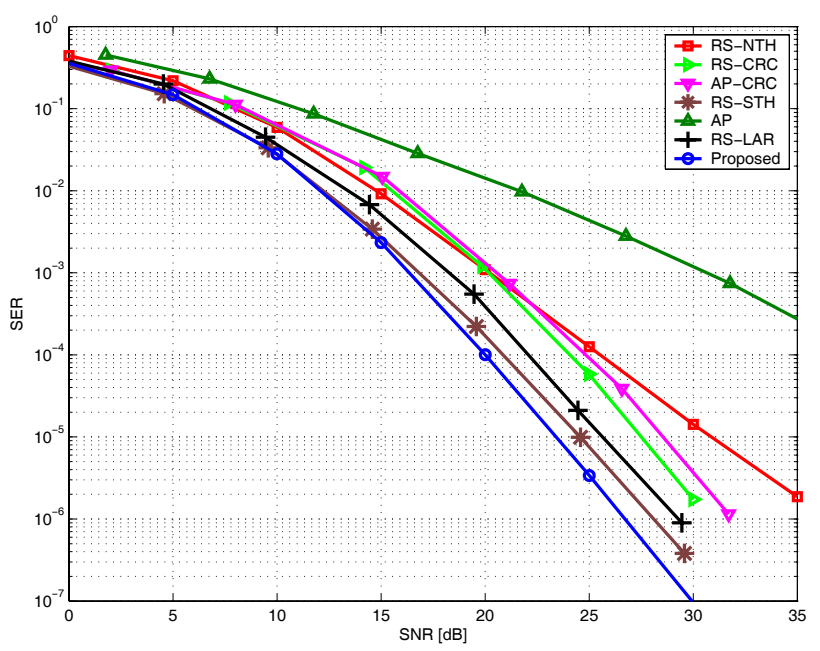

Fig. 2. SER comparison of the proposed scheme with other cooperative schemes for a channel block length of 512 symbols.

In Fig.1, we plot the SER expression given by (12) along with the simulation results. We assume equal power allocation, therefore have $K_{S}=K_{1}=K_{2}=\cdots=K_{N}=0.5$. We consider two, three, and four relays with the following geometrical gains:

- Two-relay network with $G_{S R_{i}} / G_{R_{i} D}=-30,0 \mathrm{~dB}$.

- Three-relay network with $G_{S R_{i}} / G_{R_{i} D}=-30,0,30 \mathrm{~dB}$.

- Four-relay network with $G_{S R_{i}} / G_{R_{i} D}=-30,0,30,-10$ $\mathrm{dB}$.

As observed from Fig.1, our approximate analytical expressions provide an identical match (within the thickness of the line) to the simulation results. It can be also observed that diversity orders of 3,4 , and 5 are extracted indicating the full diversity for the considered number of relays and confirming our earlier observation.

In Fig.2, we compare the performance of our proposed $\mathrm{DaF}$ multi-relay scheme (using optimum power allocation results from [1]) with other existing DaF schemes (optimized if available) in the literature. We assume two-relay network with both $G_{S R_{1}} / G_{R_{1} D}=0 \mathrm{~dB}$ and $G_{S R_{2}} / G_{R_{2} D}=0 \mathrm{~dB}$. The competing schemes are listed as: 


$$
\begin{aligned}
P_{e} & \cong \sum_{i=1}^{N} \operatorname{Pr}(i=\text { sel }) \times\left[F_{1}\left\{\Psi_{S D}\left(\alpha_{\eta_{1}}\right) \Psi_{\max }\left(\alpha_{\eta_{1}}-\Lambda_{S D}^{-1}\right)\right\}\right. \\
& +\operatorname{Pr}\left(\mu_{i}\right) F_{1}\left\{\Psi_{\max }\left(\alpha_{\eta_{1}}\right)-\Psi_{\max }\left(\alpha_{\eta_{1}}-\Lambda_{S D}^{-1}\right)\right\} \\
& +\operatorname{Pr}\left(\mu_{i}\right) F_{1}\left\{\Psi_{S D}\left(\alpha_{\eta_{1}}\right) \Psi_{R_{i} D}\left(\alpha_{\eta_{1}}\right)\left(\Psi_{\max }\left(\alpha_{\eta_{1}}\right)-\Psi_{\max }\left(\alpha_{\eta_{1}}-\Lambda_{S D}^{-1}\right)\right)\right\} \\
& -\operatorname{Pr}\left(\mu_{i}\right) F_{2}\left\{\Psi_{S D}\left(\alpha_{\eta_{1}}\right) \Psi_{R_{i} D}\left(\alpha_{\eta_{1}}\right)\left(\Psi_{\max }\left(\alpha_{\eta_{1}}+\alpha_{\eta_{2}}\right)-\Psi_{\max }\left(\alpha_{\eta_{1}}+\alpha_{\eta_{2}}-\Lambda_{S D}^{-1}\right)\right)\right\} \\
& +\operatorname{Pr}\left(\mu_{i}^{C}\right) F_{1}\left\{\Psi_{S R_{i}}\left(\alpha_{\eta_{1}}\right)\left(\Psi_{\max }\left(\alpha_{\eta_{1}}-\Lambda_{S R_{i}}^{-1}\right)-\Psi_{\max }\left(\alpha_{\eta_{1}}-\Lambda_{S D}^{-1}-\Lambda_{S R_{i}}^{-1}\right)\right)\right\} \\
& +\operatorname{Pr}\left(\mu_{i}^{C}\right) F_{1}\left\{\Psi_{S D}\left(\alpha_{\eta_{1}}\right)\left(\Psi_{\max }\left(\alpha_{\eta_{1}}\right)-\Psi_{\max }\left(\alpha_{\eta_{1}}-\Lambda_{S D}^{-1}\right)\right)\right\} \\
& \left.-\operatorname{Pr}\left(\mu_{i}^{C}\right) F_{2}\left\{\Psi_{S D}\left(\alpha_{\eta_{1}}\right) \Psi_{S R_{i}}\left(\alpha_{\eta_{2}}\right)\left(\Psi_{\max }\left(\alpha_{\eta_{1}}-\Lambda_{S R_{i}}^{-1}\right)-\Psi_{\max }\left(\alpha_{\eta_{1}}-\Lambda_{S D}^{-1}-\Lambda_{S R_{i}}^{-1}\right)\right)\right\}\right],
\end{aligned}
$$

TABLE I

DIFFERENT COOPERATION SCHEMES FOR AN N-RELAY NETWORK. "Local" CSI of a certain node is defined as the CSI of a link which terminates at that node (e.g., CSI of $\mathrm{S} \rightarrow \mathrm{R}_{i}$ is local information for ith relay). "Global" CSI describes the situation when information about all the channels is available at a certain node.

\begin{tabular}{|c|c|c|c|l|}
\hline & Diversity & CSI & Rate & Comments \\
\hline AP & Partial & Local CSI & $\frac{1}{(N+1)}$ & $\begin{array}{l}\text { Simple implementation, } \\
\text { poor performance and } \\
\text { throughput is very low. }\end{array}$ \\
\hline AP-CRC & Full & Local CSI & $\frac{1}{(N+1)}$ & $\begin{array}{l}\text { Requires CRC at relay. } \\
\text { Throughput is very low. }\end{array}$ \\
\hline RS-CRC & Full & $\begin{array}{c}\text { Global CSI } \\
\text { at D }\end{array}$ & $\frac{1}{2} \rightarrow 1$ & Requires CRC at relay. \\
\hline RS-STH & Full & $\begin{array}{c}\text { Global CSI } \\
\text { at S }\end{array}$ & $\frac{1}{2} \rightarrow 1$ & $\begin{array}{l}\text { Requires feedback } \\
\text { channel to the source. }\end{array}$ \\
\hline RS-LAR & Full & $\begin{array}{c}\text { Global CSI } \\
\text { at D }\end{array}$ & $\frac{1}{2}$ & $\begin{array}{l}\text { Requires only feed- } \\
\text { forward channel. } \\
\text { Rate is lower. }\end{array}$ \\
\hline Proposed & Full & $\begin{array}{c}\text { Global CSI } \\
\text { at D }\end{array}$ & $\frac{1}{2} \rightarrow 1$ & $\begin{array}{l}\text { Requires neither feed- } \\
\text { back CSI nor CRC. } \\
\text { Requires only feed- } \\
\text { forward CSI. (This } \\
\text { can be even avoided } \\
\text { by distributed timer } \\
\text { implementation [1]) }\end{array}$ \\
\hline
\end{tabular}

- Relay selection without any error detection or threshold (RS),

- Relay selection with 16-bit CRC in a frame length of 1024 bits (RS-CRC) [6],

- All relays participating without any error detection or threshold (AP) $)^{3}$,

- All relays participating with 16 -bit CRC in a frame length of 1024 bits (AP-CRC),

- Relay selection with static threshold (RS-STH) [8],

- Relay selection with link adaptive relaying (RS-LAR) [9].

The selection method used in RS-STH is based on the modified harmonic mean as described in [8] with optimized values of power allocation parameters. For RS-LAR, the selection criterion used is as described in [9]. In all other selection schemes, the relay selection criteria are based on (3). Table II summarizes implementation aspects of the competing cooperation schemes.

Fig.2 illustrates the performance of above cooperation schemes for a channel block length of 512 symbols. AP scheme where both relays participate without any error detec-

${ }^{3}$ This is referred as "fixed relaying" in [4].

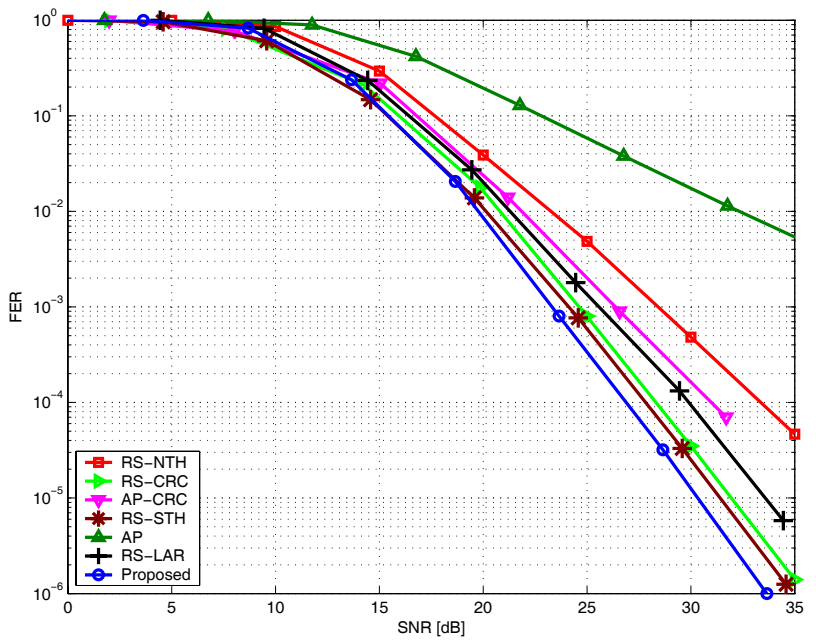

Fig. 3. SER comparison of the proposed scheme with other cooperative schemes for a channel block length of 512 symbols.

tion mechanism at relays performs the worst. For the considered relay location, it does not provide any diversity advantage. RS scheme outperforms AP and the later is not able to extract full diversity order. The use of CRC could potentially improve the performance of both AP and RS. As observed from Fig.2, both schemes with CRC (i.e., AP-CRC and RSCRC) take advantage of the full diversity and significantly outperform their counterparts without CRC. It should be noted that the implementation of RS-CRC requires maximum two time slots while AP-CRC might require more time slots (i.e., each relay with correct CRC needs an orthogonal time slot for transmission). RS-LAR performs better than two CRC-based schemes. RS-STH scheme where relay selection is performed with a static threshold is also able to outperform the RSCRC and AP-CRC schemes and avoids the use for CRC in its implementation. Our proposed scheme outperforms all previous schemes. In Fig.3, we repeat our simulation study to demonstrate frame error rate (FER) performance. Similar observations can be made for performance comparisons indicating the superiority of our scheme.

Fig.4 illustrates the performance of the above schemes for a channel block length of 128 symbols. The performance of cooperative schemes which rely only on CSI (i.e., AP, RS, RS-STH, RS-LAR as well as the proposed scheme) remain unchanged. On the other hand, performance of schemes which rely also on the accuracy of decoding at relay nodes (i.e., AP- 


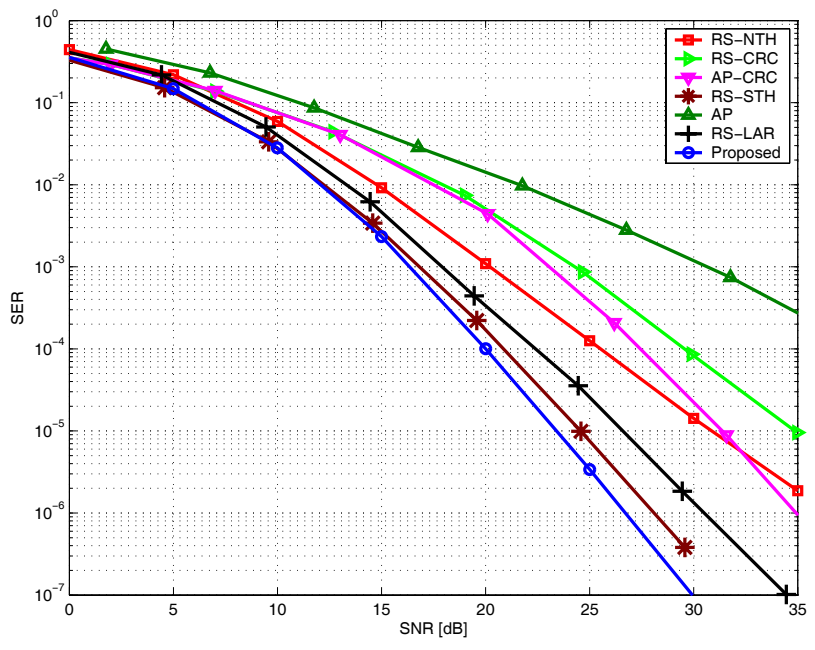

Fig. 4. SER comparison of the proposed scheme with other cooperative schemes for a channel block length of 512 symbols.

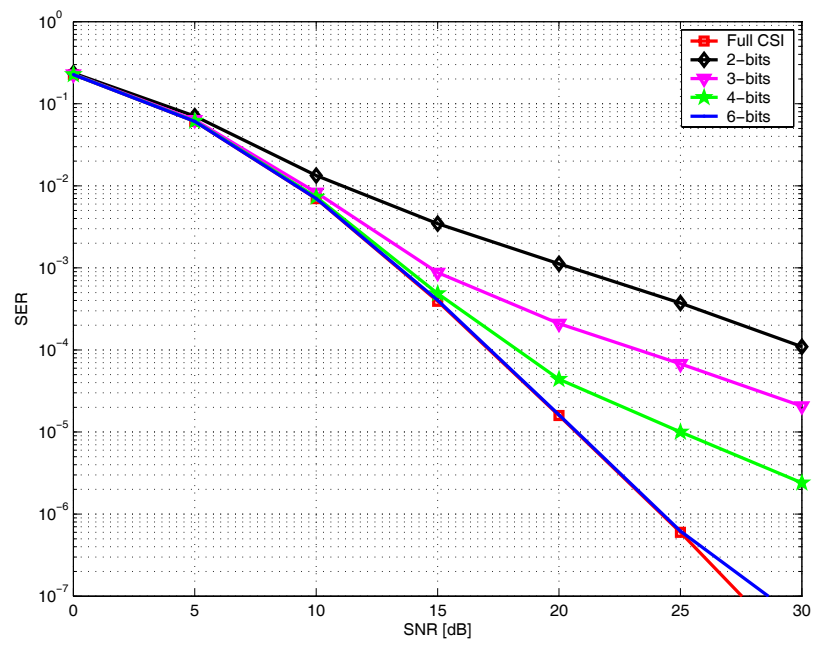

Fig. 5. Effect of $h_{S R_{i}}$ quantization on the performance of the proposed scheme.

CRC, RS-CRC) demonstrates dependency on channel block length. Particularly CRC-assisted schemes suffer a significant degradation if channel varies within CRC frame. Compared to Fig.2, we also observe from Fig.4 that the performance of AP-CRC now becomes better than that of RS-CRC.

As earlier mentioned, the proposed relay selection algorithm does not require any feedback information. It, however, requires CSI of $\mathrm{S} \rightarrow \mathrm{R}_{i}$ links at the destination node. This requires transmission of $h_{S R_{i}}$ from each relay to destination. Since the transfer of analog CSI requires to send an infinite number of bits, a control channel with limited number of feedforward bits can be used in practical implementation. To demonstrate the effect of quantization, we provide simulation results in Fig.5 where $h_{S R_{i}}$ is quantized using 2, 3, 4, and 6 bits with a non-uniform quantizer optimized for Rayleigh distributed input [13]. It is observed from Fig. 5 that as low as 6 bits would be enough to obtain a good match to the ideal case. As a final note, we would like to point out that this feedforward channel can be also avoided if one prefers a distributed implementation of relay selection algorithm similar to [6]. The description of such an implementation can be found in [1].

\section{CONCLUSION}

In this letter, we have proposed a simple relay selection method for multi-relay networks with $\mathrm{DaF}$ relaying. The proposed method avoids the use of error detection methods at relay nodes and does not require close-loop implementation with feedback information to the source. Its implementation however requires channel state information of source-to-relay channels at the destination. This can be easily done in practice through a feedforward channel from the relay to the destination. Our SER performance analysis has shown that the proposed relay-selection method is able to extract the full diversity. Our simulation results have further demonstrated the superior performance of the proposed scheme over its competitors.

\section{APPENDIX A}

In this appendix, we calculate marginal pdf of $\lambda_{\max }$ and joint pdf of $\lambda_{S R_{s e l}}$ and $\lambda_{R_{s e l} D}$ which are required to take expectation of (9).

Let us define $\lambda_{i}=\min \left(\lambda_{S R_{i}}, \lambda_{R_{i} D}\right)$. Under the Rayleigh fading assumption, both $\lambda_{S R_{i}}$ and $\lambda_{R_{i} D}$ follow exponential distribution. Recall that $\Delta$ denotes the set of permutations of $1,2, \ldots, N, P(\sigma)$ denotes the probability of one particular permutation $\sigma \in \Delta$, and $\lambda_{(i)}$ denotes the ordered sequence of $\lambda_{i}$ s, i.e., $\lambda_{(1)}>\lambda_{(2)}>\lambda_{(3)}>\ldots>\lambda_{(N)}$. It can be shown [14] that we can transform $\lambda_{(i)} \mathrm{s}$ into a set of new conditionally independent variables $V_{n}$ such that $\lambda_{(i)}=\sum_{n=i}^{N} A_{n} V_{n}$ where $A_{n}=\left(\sum_{m=1}^{n} \Lambda_{m}^{-1}\right)^{-1}$. The joint pdf of $V_{n}$ is given by where $\operatorname{Pr}\{\sigma\}=\prod_{k=1}^{N} \Gamma_{\sigma_{k}}^{-1}\left(\sum_{m=1}^{k} \Gamma_{\sigma_{m}}^{-1}\right)^{-1}$ and $f_{\mathbf{V}}\left(\left\{V_{n}\right\}_{n=1}^{N}\right)=$ $\sum_{\sigma \in S} \operatorname{Pr}\{\sigma\} f_{\mathbf{V} \mid \sigma}\left(\left\{V_{n}\right\}_{n=1}^{N} \mid \sigma\right)$ for $\left[0<V_{n}<\infty\right]$ with $\Gamma_{n}=\left(\sum_{m=1}^{n} \Lambda_{m}^{-1}\right)\left(\sum_{m=1}^{n} \Lambda_{\sigma_{m}}^{-1}\right)^{-1}$. The above transformation of variables enables us to find the moment generating function (MGF) of variable $\lambda_{\max }=\lambda_{(1)}=\sum_{n=1}^{N} A_{n} V_{n}$ as $\Psi_{\max }(s \mid \sigma)=\prod_{n=1}^{N}\left(1+s A_{n} \Gamma_{n}\right)^{-1}$.

For probabilities of event $\xi_{i}$ and $\xi_{i}^{C}$, we first define $\Delta_{i} \subset \Delta$ as the set of all the permutations for which $i$ is the first element. Then we have $\operatorname{Pr}(i=s e l)=\sum_{\sigma \in \Delta_{i}} \operatorname{Pr}(\sigma)$, which denotes the probability of $i$ th relay node being selected. Recall that $\mu_{i}$ denotes the event of $\lambda_{S R_{i}}<\lambda_{R_{i} D}$ and $\mu_{i}^{C}$ its complementary event, i.e., $\lambda_{S R_{i}}>\lambda_{R_{i} D}$. The probabilities of these two events can be approximated ${ }^{4}$ as $\operatorname{Pr}\left(\xi_{i}\right) \cong$ $\operatorname{Pr}(i=$ sel $) \operatorname{Pr}\left(\mu_{i}\right)$, and $\operatorname{Pr}\left(\xi_{i}^{C}\right) \cong \operatorname{Pr}(i=$ sel $) \operatorname{Pr}\left(\mu_{i}^{C}\right)$ with $\operatorname{Pr}\left(\mu_{i}\right)=\Lambda_{R_{i} D} /\left(\Lambda_{S R_{i}}+\Lambda_{R_{i} D}\right)$ and $\operatorname{Pr}\left(\mu_{i}^{C}\right)=$ $\Lambda_{S R_{i}} /\left(\Lambda_{S R_{i}}+\Lambda_{R_{i} D}\right)$. Here, $\Lambda_{R_{i} D}$ and $\Lambda_{S R_{i}}$ are the average values of received SNRs and are given by $\Lambda_{R_{i} D}=E\left[\lambda_{R_{i} D}\right]=$ $G_{R_{i} D} K_{i}\left(E / N_{0}\right)$ and $\Lambda_{S R_{i} D}=E\left[\lambda_{S R_{i}}\right]=G_{S R_{i}} K_{S}\left(E / N_{0}\right)$.

Now let us calculate the joint pdf of $\lambda_{S R_{s e l}}$ and $\lambda_{R_{s e l} D}$ conditioned on the respective events $\xi_{i}$ and $\xi_{i}^{C}$. For $\xi_{i}$, we have $\lambda_{S R_{i}}=\lambda_{\max }$, thus conditional statistics of $\lambda_{S R_{\text {sel }}}$ and $\lambda_{R_{s e l} D}$ are given by $M G F\left(\lambda_{S R_{s e l}}\right)=M G F\left(\lambda_{\max }\right)$ and

$$
f_{R_{s e l} D}\left(\lambda \mid \xi_{i}\right)=\left\{\begin{array}{cc}
\left(\frac{1}{\Lambda_{R_{i} D}}\right) \exp \left(-\frac{\lambda}{\Lambda_{R_{i} D}}\right), & \lambda \geq \lambda_{\max } \\
0, & \text { otherwise }
\end{array}\right.
$$

\footnotetext{
${ }^{4}$ Exact value for event $\xi_{i}$ can be calculated as $\operatorname{Pr}\left(\xi_{i}\right)=$ $\operatorname{Pr}\left(\lambda_{R_{i} D}>\lambda_{S R_{i}}>\lambda_{k}, \forall k \neq i\right)$
} 


$$
\begin{array}{r}
\Phi_{2}^{C}=F_{1}\left(\Psi_{S D}\left(\alpha_{\eta_{1}}\right)\left[\Psi_{\max }\left(\alpha_{\eta_{1}}\right)-\Psi_{\max }\left(\alpha_{\eta_{1}}-\left(\Lambda_{S D}^{-1}\right)\right)\right]\right) \\
-F_{2}\left(\Psi _ { S D } ( \alpha _ { \eta _ { 1 } } ) \Psi _ { S R _ { i } } ( \alpha _ { \eta _ { 2 } } ) \left[\Psi_{\max }\left(\alpha_{\eta_{1}}-\left(\Lambda_{S R_{i}}^{-1}\right)\right)\right.\right. \\
\left.\left.-\Psi_{\max }\left(\alpha_{\eta_{1}}-\left(\Lambda_{S R_{i}}^{-1}\right)-\left(\Lambda_{S D}^{-1}\right)\right)\right]\right) .
\end{array}
$$

For event $\xi_{i}^{C}$, we have $\lambda_{R_{i} D}=\lambda_{\max }$. Thus conditional statistics of $\lambda_{R_{s e l} D}$ and $\lambda_{S R_{s e l}}$ are obtained respectively as $M G F\left(\lambda_{R_{s e l} D}\right)=M G F\left(\lambda_{\max }\right)$ and

$$
f_{S R_{s e l}}\left(\lambda \mid \xi_{i}^{C}\right)=\left\{\begin{array}{cc}
\left(\frac{1}{\Lambda_{S R_{i}}}\right) \exp \left(-\frac{\lambda}{\Lambda_{S R_{i}}}\right), & \lambda \geq \lambda_{\max } \\
0, & \text { otherwise }
\end{array}\right.
$$

\section{APPENDIX B}

In this appendix, we calculate $\Phi_{0}, \Phi_{k}$ and $\Phi_{k}^{C}, k=1,2$ which appear in (11). Noting $\lambda_{S D}$ follows an exponential distribution under the considered Rayleigh fading assumption, we have $\Phi_{0}=E_{\lambda}\left[T_{0}\right]=E_{\lambda_{\max }}\left[\int_{\lambda_{\max }}^{\infty} \beta(\lambda) f_{\lambda_{S D}}(\lambda) d \lambda\right]$. Using the definition of $\beta$ (.) from (7), we have

$$
\Phi_{0}=F_{1}\left\{\Psi_{S D}\left(\alpha_{\eta_{1}}\right) E_{\lambda_{\max }}\left[\exp \left(-\lambda_{\max }\left(\Lambda_{S D}^{-1}-\alpha_{\eta_{1}}\right)\right)\right]\right\}
$$

where $\alpha_{\eta}$ and $F_{1}\{$.$\} are earlier defined by (13). Averaging$ over $\lambda_{\max }$ yields

$$
\Phi_{0}=F_{1}\left\{\Psi_{S D}\left(\alpha_{\eta_{1}}\right) \Psi_{\max }\left(\alpha_{\eta_{1}}-\Lambda_{S D}^{-1}\right)\right\}
$$

where $\Phi_{S D}($.$) and \Phi_{\max }($.$) are MGFs of \lambda_{S D}$ and $\lambda_{\max }$. For the calculations of $\Phi_{k}$ and $\Phi_{k}^{C}, k=1,2$, we need to consider events $\xi_{i}$ and $\xi_{i}^{C}$.

\section{A. Case I (Event $\left.\xi_{i}\right)$}

From (9), we have $T_{1}=P\left(\lambda_{S D}<\lambda_{\max }\right) \beta\left(\lambda_{\max }\right)$ for this event. Taking expectation with respect to $\lambda_{S D}$, we have $E_{\lambda_{S D}}\left[T_{1}\right]=\left(1-\exp \left(-\lambda_{\max } \Lambda_{S D}\right)\right) \beta\left(\lambda_{\max }\right)$. Using the definition of $\beta($.$) and further taking expectation with respect$ to $\lambda_{\max }$, we obtain

$$
\Phi_{1}=E_{\lambda}\left[T_{1}\right]=F_{1}\left(\Psi_{\max }\left(\alpha_{\eta_{1}}\right)-\Psi_{\max }\left(\alpha_{\eta_{1}}-\Lambda_{S D}^{-1}\right)\right) .
$$

On the other hand, $T_{2}$ is given as $T_{2}=$ $P\left(\lambda_{S D}<\lambda_{\max }\right)\left[1-\beta\left(\lambda_{S R_{s e l}}\right)\right] \beta\left(\lambda_{R_{s e l} D}+\lambda_{S D}\right)$. First taking expectation with respect to $\lambda_{S D}$ and $\lambda_{R_{s e l} D}$ using pdf given by (16) and then averaging over $\lambda_{\max }$, we have

$$
\begin{array}{r}
\Phi_{2}=F_{1}\left(\Psi _ { S D } ( \alpha _ { \eta _ { 1 } } ) \Psi _ { R _ { i } D } ( \alpha _ { \eta _ { 1 } } ) \left[\Psi_{\max }\left(\alpha_{\eta_{1}}-\Lambda_{R_{i} D}^{-1}\right)\right.\right. \\
\left.\left.-\Psi_{\max }\left(\alpha_{\eta_{1}}-\Lambda_{R_{i} D}^{-1}-\Lambda_{S D}^{-1}\right)\right]\right) \\
-F_{2}\left(\Psi _ { S D } ( \alpha _ { \eta _ { 1 } } ) \Psi _ { R _ { i } D } ( \alpha _ { \eta _ { 1 } } ) \left[\Psi_{\max }\left(\alpha_{\eta_{1}}+\alpha_{\eta_{2}}-\Lambda_{R_{i} D}^{-1}\right)\right.\right. \\
\left.\left.-\Psi_{\max }\left(\alpha_{\eta_{1}}+\alpha_{\eta_{2}}-\Lambda_{R_{i} D}^{-1}-\Lambda_{S D}^{-1}\right)\right]\right) .
\end{array}
$$

\section{B. Case II (Event $\xi_{i}^{C}$ )}

From (9), we have $T_{1}=P\left(\lambda_{S D}<\lambda_{\max }\right) \beta\left(\lambda_{S R_{s e l}}\right)$ for this event. Averaging over $\lambda_{S D}$, we obtain $E_{\lambda_{S D}}\left[T_{1}\right]=$ $\left(1-\exp \left(-\lambda_{\max } / \Lambda_{S D}\right)\right) \beta\left(\lambda_{S R_{s e l}}\right)$. Using the definition of $\beta($.$) and taking expectations over \lambda_{S R_{s e l}}$ and $\lambda_{\max }$, we obtain

$$
\begin{aligned}
\Phi_{1}^{C}=E_{\lambda}\left[T_{1}\right]=F_{1} & \left(\Psi _ { S R _ { i } } ( \alpha _ { \eta _ { 1 } } ) \left[\Psi_{\max }\left(\alpha_{\eta_{1}}-\Lambda_{S R_{i}}^{-1}\right)\right.\right. \\
& \left.\left.-\Psi_{\max }\left(\alpha_{\eta_{1}}-\Lambda_{S R_{i}}^{-1}-\Lambda_{S D}^{-1}\right)\right]\right) .
\end{aligned}
$$

On the other hand, we have $T_{2}=$ $P\left(\lambda_{S D}<\lambda_{\max }\right)\left[1-\beta\left(\lambda_{S R_{s e l}}\right)\right] \beta\left(\lambda_{R_{s e l} D}+\lambda_{S D}\right)$.

Inserting $\beta($.$) in T_{2}$, taking expectation of the resulting expression with respect to $\lambda_{S R_{s e l}}$ and $\lambda_{\max }$, we obtain (23).

\section{REFERENCES}

[1] M. M. Fareed and M. Uysal, "A novel relay selection method for decodeand-forward relaying," in IEEE Canadian Conference Electrical Computer Engineering 2008 (CCECE'08), Niagara Falls, Ontario, Canada, May 2008.

[2] A. Sendonaris, E. Erkip, and B. Aazhang, "User cooperation diversity Part I: System description," IEEE Trans. Commun., vol. 51, no. 11, pp. 1927-1938, Nov. 2003.

[3] - "User cooperation diversity, part II: implementation aspects and performance analysis," IEEE Trans. Commun., vol. 51, no. 11, pp. 19391948, Nov. 2003

[4] J. N. Laneman, D. N. C. Tse, and G. W. Wornell, "Cooperative diversity in wireless networks: efficient protocols and outage behavior," IEEE Trans. Inform. Theory, vol. 50, no. 12, pp. 3062-3080, Dec. 2004.

[5] T. Wang, A. Cano, G. B. Giannakis, and J. N. Laneman, "Highperformance cooperative demodulation with decode-and-forward relays," IEEE Trans. Commun., vol. 55, no. 7, pp. 1427-1438, 2007.

[6] A. Bletsas, A. Khisti, D. P. Reed, and A. Lippman, "A simple cooperative diversity method based on network path selection," IEEE J. Select. Areas Commun., vol. 24, no. 3, pp. 659-672, 2006.

[7] E. Beres and R. Adve, "Selection cooperation in multi-source cooperative networks," IEEE Trans. Wireless Commun., vol. 7, no. 1, pp. $118-127,2008$

[8] A. S. Ibrahim, A. K. Sadek, W. Su, and K. J. R. Liu, "Relay selection in multi-node cooperative communications: when to cooperate and whom to cooperate with?" in Proc. IEEE GLOBECOM 2006.

[9] Z. Yi and I.-M. Kim, "Diversity order analysis of the decode-andforward cooperative networks with relay selection," IEEE Trans. Wireless Commun., vol. 7, no. 5-2, pp. 1792-1799, 2008.

[10] T. Wang, R. Wang, and G. B. Giannakis, "Smart regenerative relays for link-adaptive cooperative communications," in 40th Conf. Inform. Sciences Syst. (CISS'06), Mar. 2006.

[11] H. Ochiai, P. Mitran, and V. Tarokh, "Variable-rate two-phase collaborative communications protocols for wireless networks," IEEE Trans. Inform. Theory, vol. 52, no. 9, pp. 4299-4312, Sept. 2006.

[12] M. K. Simon and M. S. Alouini, Digital Communication over Fading Channel: A Unified Approach to Performance Analysis. New York: Wiley, 2000.

[13] W. A. Pearlman and G. H. Senge, "Optimal quantization of the rayleigh probability distribution," IEEE Trans. Commun., vol. 27, no. 1, pp. 101$112,1979$.

[14] M. Z. Win and J. H. Winters, "Analysis of hybrid selection/maximalratio combining of diversity branches with unequal snr in rayleigh fading," in Proc. 49th IEEE VTC, 1999. 